\title{
Exposición infantil a plastificantes potencialmente tóxicos en productos de uso oral
}

\author{
Lilia Patricia Bustamante-Montes, MC, M en C, ${ }^{(1,3)}$ Beatriz Lizama-Soberanis, IQ , M en Admon, D ra en C, (1) \\ Flavio Vázquez-Moreno IQ , Dr en Fisicoquímica Mol, (1,4) \\ María Magdalena García-Fábila, IQ , M en C, ${ }^{(1)}$ Kira Susana Corea-Téllez, IQ , M en C, ${ }^{(1)}$ \\ Gustavo 0 laiz-Fernández, MC, MSP, ${ }^{(2,3)}$ Víctor Hugo Borja-A burto, MC, PhD. ${ }^{(2,5)}$
}

\begin{abstract}
Bustamante-Montes LP, Lizama-Soberanis B, Vázquez-Moreno F, García-Fábila MM, Corea-Téllez KS, Olaiz-Fernández G, Borja-Aburto VH. Exposición infantil a plastificantes potencialmente tóxicos en productos de uso oral. Salud Publica Mex 2004;46:501-508.

El texto completo en inglés de este artículo está disponible en: http://www.insp.mx/salud/46/eng
\end{abstract}

\begin{abstract}
Resumen
Objetivo. Determinar la prevalencia en el uso de productos infantiles orales entre menores de tres años de edad y medir su concentración de ftalatos, sustancias potencialmente tóxicas. Material y métodos Se realizó, en 1999, una entrevista domiciliaria a 199 madres de niños del área metropolitana de la ciudad de Toluca. Por cromatografía de gases se identificaron y cuantificaron diversos ftalatos de productos de uso oral empleados por los niños participantes y se estimó la contribución de estas fuentes a la ingesta diaria de ftalatos. Resultados La prevalencia de uso de estos productos fue de $13 \%$, siendo mayor entre los niños, menores de 18 meses de edad, pertenecientes al estrato socioeconómico bajo. Las concentraciones variaron desde trazas hasta $67.0 \%$ del peso. La exposición media calculada proveniente de los productos manufacturados con policloruro de vinilo y ftalatos fue de $13.94 \mu \mathrm{g} / \mathrm{kg}$ de peso/día, IC $95 \%$ $(9.08,18.89)$. Conclusiones La exposición a ftalatos proveniente de productos para chupar o morder se encuentra dentro de los límites reportados en otros países; sin embar-
\end{abstract}

\author{
Bustamante-Montes LP, Lizama-Soberanis B, \\ Vázquez-Moreno F, García-Fábila MM, Corea-Téllez KS, \\ Olaiz-Fernández G, Borja-Aburto VH. \\ Infant exposure to potentially \\ toxic plasticizers in products for oral use. \\ Salud Publica Mex 2004;46:501-508. \\ The English version of this paper \\ is available at: http://www.insp.mx/salud/46/eng
}

\section{Summary}

Objective To estimate the prevalence of oral product use in children less than three years of age, and to measure the concentration of phthalates as potentially toxic products. Material and Methods. In 1999, 199 mothers of children living in the city of Toluca agreed to household interviews. Samples of oral products used by the children were taken and analyzed by gas chromato graphy to identify and quantify phthalate concentrations, to estimate the daily intake of phthalates from this source. Results The prevalence of oral product use was 13\%. Male infants less than 18 months of age of low socioeconomic level used them more frequently. The concentrations ranged from traces to $67 \%$ weight. The mean exposure to products manufactured with polyvinyl chloride and phthalates was $13.94 \mu \mathrm{g} / \mathrm{kg} / \mathrm{day}(95 \% \mathrm{Cl} 9.08$, 18.89). Conclusions The daily dose of phthalate intake from products for infants to suck or bite did not exceed the recommended limit established in other countries. N evertheless, other so urces can contribute to increase the total dose. Since some phthalates are harmful to the reproductive

Proyecto financiado por la Universidad Autónoma del Estado de México, proyecto 1384/99; Consejo Nacional de Ciencia y Tecnología, proyecto 3089-2; Instituto $\mathrm{N}$ acional de Salud Pública, proyecto: Ftalatos y efectos a la salud, y D irección General de Salud Ambiental, Programa de Investigación: Ftalatos y efectos a la salud.

(1) Universidad Autónoma del Estado de México.Toluca, Estado de México, México.

(2) Dirección General de Salud A mbiental, Secretaría de Salud. México, D F, México.

(3) Instituto $N$ acional de Salud Pública. Cuernavaca, Morelos, México.

(4) Instituto Mexicano del Petróleo.

(5) Instituto Mexicano del Seguro Social.

Fecha de recibido: 10 de febrero de 2004 • Fecha de aprobado: 21 de septiembre de 2004

Solicitud de sobretiros: Dr.Víctor Hugo Borja Aburto. Centro Médico N acional Siglo XXI.Avenida Cuauhtémoc 330, Edificio C 5o piso, colonia Doctores, Delegación Cuauhtémoc 06725, México, DF, México. Correo electrónico: victor.borja@ imss.gob.mx 
go, otras fuentes pueden incrementarla. D ado que algunos ftalatos han mostrado ser tóxicos en el sistema reproductivo, y este potencial efecto es plausible en el hombre, es necesaria la investigación de otras fuentes y determinar la exposición total a través de biomarcadores. El texto completo en inglés de este artículo está disponible en: http:// www.insp.mx/salud/46/eng

Palabras clave: ftalatos; exposición; policloruro de vinilo (PCV); México system in animals and this potential effect may also be expected in humans, it is necessary to assess other sources and determine exposure using biomarkers. The English version of this paper is available at: http://www.insp.mx/salud/ 46/eng

Key words: phthalate; exposure; polyvinyl chloride (CPV); Mexico os ftalatos son plastificantes que dan flexibilidad

y durabilidad a productos del policloruro de vinilo (PCV), y su concentración en los artículos ha sido en general de $50 \%$ en adelante del peso final del producto, de acuerdo con la flexibilidad que éste requiere. Como los plastificantes no son polimerizados dentro de la matriz del plástico, pueden desprenderse con el tiempo y el uso, y liberarse al ambiente, momento en el que puede ocurrir la exposición en los humanos, especialmente al ponerse en contacto con sustancias lipofílicas. Estos ésteres del ácido ftálico son considerados como contaminantes ubicuos en alimentos, aire, suelo y sedimentos. Los ftalatos se bioacumulan en invertebrados, peces y plantas, pero no se biomagnifican porque los animales superiores metabolizan y excretan los ftalatos eficientemente. ${ }^{1}$

La potencialidad tóxica para los humanos fue manejada como baja hasta 1982, cuando el informe del Programa Nacional de Toxicología de los Estados Unidos de América (EUA) (NTP, por sus siglas en inglés), reportó carcinogenecidad en roedores. ${ }^{1,2}$ Actualmente esta posibilidad de efectos carcinogénicos se ha minimizado para el humano. Sin embargo, recientemente se han observado otros efectos, como la disrupción endocrina masculina en animales de laboratorio y en algunos ecosistemas, debido a su acción antiandrogénica. ${ }^{3-5}$

Los aditivos plastificantes del PCV han sido utilizados ampliamente en la industria de los juguetes, principalmente en la manufactura de juguetes blandos, de manera que los niños pueden estar altamente expuestos a estas sustancias. Greenpeace, en 1997, ${ }^{6}$ mostró que varios productos blandos destinados a ser chupados o mordidos por niños contienen entre $10 \mathrm{y}$ $40 \%$ de ftalatos. Otros estudios también han demostrado el alto contenido de ftalatos en los juguetes. ${ }^{7}$

Como medida precautoria y mientras se realizan los estudios necesarios, la industria juguetera de EUA y Canadá retiró voluntariamente los ftalatos de la manufactura de estos productos infantiles de uso oral.
Entre las necesidades de investigación identificadas en este programa se encuentran la determinación potencial del riesgo para los humanos, incluyendo las dosis cancerígenas, el tiempo que los niños chupan o mastican productos que puedan contener ftalatos y la cantidad de ftalatos liberados de los productos para niños. $^{8}$

Este reporte presenta los resultados de un estudio que investigó los patrones de uso de estos productos y la estimación de la ingesta diaria de ftalatos por parte de una población de niños mexicanos.

\section{Material y métodos}

Para evaluar la exposición potencial a ftalatos por el uso de productos de uso oral en niños se realizó un estudio transversal, el cual se desarrolló en tres fases diferentes. La primera fase consistió en una encuesta para evaluar la frecuencia de uso de estos objetos y obtención de muestras de los productos usados; la segunda fase se enfocó a determinar la cantidad de ftalatos contenidos en las muestras obtenidas y la tercera fase en determinar la ingesta diaria a partir del contenido y migración de los plastificantes en los productos empleados.

La encuesta se realizó en la ciudad de Toluca en los meses de junio y julio de 1999. La muestra fue seleccionada de manera aleatoria estratificada a partir de un listado de hogares donde viven niños menores de tres años de edad, proporcionado por el Instituto de Salud del Estado de México (ISEM), y elaborado a partir de los censos de vacunación. El número diferente de niños por estrato se debe a que el tipo de muestreo fue estratificado y proporcional al tamaño del estrato de niños menores de tres años de edad listados por el ISEM, los cuales fueron 30, 4809 y 2593 para los estratos socioeconómicos alto (I), medio (II) y bajo (III), respectivamente. La muestra fue incrementada $35 \%$ a cada estrato debido a que se desconocía el porcentaje 
de respuesta y dificultades para la localización de la población a encuestar. En resumen, de un total de 7432 niños, se seleccionaron 74 de estrato socioeconómico bajo, 122 de estrato socioeconómico medio y tres del alto.

Una vez localizadas las madres de niños menores de tres años de edad, se les invitó a participar en el estudio, previo consentimiento informado. Se les aplicó un cuestionario estructurado que incluyó preguntas sobre disponibilidad y uso de los productos motivo del estudio, considerando la frecuencia y tiempo de su uso. Los niños fueron pesados con básculas Salter. Al finalizar la entrevista se solicitó a la madre que mostrara el producto de uso oral o juguete de mayor empleo por el niño, con el propósito de verificar el uso del mismo, y se le propuso el cambio del producto usado por un nuevo producto libre de ftalatos (baberos, jabones, cepillos de dientes y esponjas).

El producto obtenido fue analizado en el laboratorio de química instrumental, de la Facultad de Química de la Universidad Autónoma del Estado de México (UAEM), con la siguiente metodología general para la identificación y cuantificación de ftalatos en PCV plastificado: primero se establecieron las condiciones óptimas de operación del equipo de laboratorio, que consistieron principalmente en determinar el tiempo de retención de cada estándar de ftalato y en elaborar la curva de calibración con la mezcla estándar de los ésteres ftálicos. Un segundo paso consistió en montar y validar el método, utilizando plastificados de PCV mediante muestras preparadas en el laboratorio de polímeros con concentraciones conocidas de PCV-ftalatos con rangos de composición en peso de 80:20, 70:30, 60:40, 50:50, 40:60 y 20:80, respectivamente; (por ejemplo, 80:20, significa $80 \%$ de PCV y 20\% de ftalato). Cabe señalar que las muestras plastificadas se prepararon con cada uno de los ftalatos. Se procedió a extraer los ftalatos de los plastificados preparados con hexano por 12 horas, este tiempo se decidió debido a la deficiente homogeneización de los plastificantes en el PCV. Posterior a la validación del método, se inyectó el extracto en el cromatógrafo de gases para su lectura. Finalmente, se aplicó el método validado a las muestras obtenidas en la encuesta, las cuales habían sido revisadas previamente mediante análisis infrarrojo para determinar si contenían PCV.

La cantidad y velocidad de migración del ftalato de bis-2(etilohexilo), el ftalato más frecuentemente encontrado en los productos, fue estimado en saliva de voluntarios adultos a partir de PCV plastificado en una concentración 40/60 (PCV/DEHP), estas concentraciones corresponden al escenario de mayor riesgo. La difusión fue calculada mediante la teoría de contribución por grupos y experimentación dinámica en saliva de voluntarios. ${ }^{9}$ La descripción completa del método para calcular la difusión de ftalatos se presenta en otro reporte: ${ }^{10}$ brevemente se describe: de artículos comerciales de uso oral se efectuaron tres cortes circulares que aproximaron un área de $10 \mathrm{~cm}^{2}$ y que contenían ftalato de bis(2-etil hexilo) en un $60 \%$ en peso. Cada porción se le proporcionó a voluntarios masculinos para que fueran chupados durante 30 minutos. Se les pidió que retuvieran la saliva generada en el proceso y se colectaron $5 \mathrm{ml}$ de saliva, de los cuales fue extraído el ftalato mediante solventes. Esta parte del proceso fue realizado por duplicado. Se prepararon los estándares internos y se analizó mediante cromatografía de gases y detector de FID (ionización de flama) inyectándose $1 \mu \mathrm{l}$ cada vez, y analizándose por triplicado. Con las concentraciones de DEHP en saliva humana obtenidas de los experimentos se calculó en $\mu \mathrm{g} / \mathrm{cm}^{2} / \mathrm{h}$ la difusión o migración. ${ }^{10}$

La difusión e ingesta diaria fueron estimadas con las siguientes fórmulas:

Difusión $\left(\mu \mathrm{g} / \mathrm{cm}^{2} / \mathrm{h}\right)=(\mathrm{DEHP})(\mathrm{V}) /(\mathrm{A})(\mathrm{t})$

Dosis $(\mu \mathrm{g} / \mathrm{kg} /$ día $)=($ Difusión) $(24) /(\mathrm{p})$

donde:

$\mathrm{DEHP}=$ concentración del final de di(2etylhoxyl)phtalate en saliva de voluntarios humanos, $\mu \mathrm{g} / \mathrm{cm}^{3}$ al tiempo t y distancia x (media 1.51, DE 0.27) obtenida experimentalmente y contrastada con el modelo matemático.

$\mathrm{V}=$ volumen de la cavidad bucal $20 \mathrm{~cm}^{3}$ de acuerdo con el Comité Científico de Toxicidad, Ecotoxicidad y Ambiente de los EUA (CSTEE), por sus siglas en inglés. ${ }^{11}$

$\mathrm{A}=$ área superficial del artículo, en $10 \mathrm{~cm}^{2}$ de acuerdo con CSTEE. ${ }^{11}$

$\mathrm{t}=$ tiempo, horas de chupado de acuerdo a los resultados de la encuesta (0.5).

$\mathrm{p}=$ peso corporal promedio de los niños de acuerdo a los resultados de la encuesta en $\mathrm{kg}$ (10.366).

\section{Resultados}

Se aplicaron un total de 199 encuestas a mamás de niños menores de tres años de edad. No hubo pérdidas ni renuencia a colaborar en el estudio. El 2.0\% de las encuestas pertenece a niños del estrato socioeconómico alto (I); $62.8 \%$ al estrato socioeconómico medio (II) y $35.2 \%$ al estrato socioeconómico bajo (III). El $48.2 \%$ de las encuestas correspondieron a niñas y $51.8 \%$ a niños. El promedio de edad fue de 18 meses. La media del peso de todos los niños fue de $10.6 \mathrm{Kg}$ con un intervalo entre los 3.0 y $18.5 \mathrm{~kg}$. 
La media de edad de las madres de estos niños fue de 28 años, con un rango entre los 15 y 43 años. En cuanto a la escolaridad la media fue de 11 años de estudio completos. El 77\% de las madres se encargaban directamente del cuidado de sus hijos y $9 \%$ los enviaban a guarderías.

El 82\% de las madres reportó que sus hijos empleaban productos infantiles de uso oral. $\mathrm{Al}$ interior de los estratos entre los niños que usan estos productos se observaron diferencias. Los niños del estrato I y II utilizaron los productos en un $78.3 \%$ y los niños del estrato III en $90 \%$, $\left(\chi^{2}=4.3, p=0.04\right)$

El $80 \%$ de las mamás que afirmaron que sus hijos usaban en el momento de la encuesta productos infantiles de uso oral entregaron alguno de los productos de uso actual para ser analizado en el laboratorio. En el cuadro I se presentan los productos obtenidos durante la encuesta. En relación con los niños que usan productos de mayor riesgo de contener ftalatos de acuerdo con la literatura como son los chupones y mordederas, se encontró una prevalencia de uso de $13.0 \%$. Del total de productos obtenidos en la encuesta se encontró $11.4 \%$ de productos fabricados con PCV plastificados principalmente con DEHP; el tipo de productos fue principalmente mamilas de biberones, utilizados en la alimentación de los niños.

Los niños usaban en promedio 30 minutos al día los productos de uso oral que fueron entregados para su análisis. Al interior de los estratos encontramos diferencias estadísticamente significativas $(p=0.04)$; los niños del estrato II tienen en promedio 18 minutos menos tiempo estos productos en la boca, en contraste con los otros estratos, y los niños del estrato III tienen aproximadamente 20 minutos más estos productos en la boca, comparados con los otros dos estratos. En gene-

\section{Cuadro I \\ TIPOS DE PRODUCTOS QUE USABAN LOS NIÑOS en el momento de La encuesta.Toluca, México, 1999}

\begin{tabular}{lrr}
\multirow{2}{*}{ Tipo de producto } & \multicolumn{2}{c}{ Frecuencia } \\
\cline { 2 - 3 } Mamilas & $\mathrm{N} 0$. & $\%$ \\
\hline Biberones & 103 & 63.6 \\
\hline Vasos entrenadores & 15 & 9.3 \\
\hline Chupones o pacificadores & 26 & 16.0 \\
\hline Mordederas & 13 & 8.0 \\
\hline Juguetes & 3 & 1.9 \\
\hline Otro & 1 & 0.6 \\
\hline Total & 1 & 0.6 \\
\hline
\end{tabular}

ral, $86.4 \%$ de los niños chupaban los productos menos de una hora al día.

Se indagó sobre otras posibles fuentes de exposición a productos plásticos y potencialmente a ftalatos, entre las que sobresalieron el uso de utensilios de plástico para comer (cuadro II).

Como puede observarse en el cuadro III, la frecuencia en el uso de productos infantiles de uso oral fue semejante en los distintos estratos de edad, escolaridad y ocupación de la madre, con quien cuidaba al niño y con los antecedentes de lactancia. La prevalencia de lactancia materna durante la encuesta fue de $26.2 \%$ y entre los niños que no lactaban en ese momento $63.8 \%$ lo hizo en el pasado. Entre los niños que habían lactado casi $70.0 \%$ pertenecía al estrato socioeconómico medio comparado con $29.0 \%$ del estrato socioeconómico bajo; la diferencia fue estadísticamente significativa $(p=0.04)$. La frecuencia de uso fue mayor entre los niños menores, sobre todo en los menores de 18 meses de edad y fue mayor entre los niños que en las niñas, así como en el estrato socioeconómico bajo o más vulnerable. Finalmente, se construyó un modelo de regresión logística para la variable resultado uso de productos orales donde se consideraron las variables que en el análisis bivariado mostraron diferencias estadísticamente significativas o eran biológicamente plausibles. El modelo final mantuvo a la edad menor de 18 meses, género masculino y estrato socioeconómico bajo como variables asociadas con el uso de los productos motivo del estudio.

Los materiales más utilizados en la fabricación de los productos examinados fueron: silicona, polipropileno, polietileno, $\mathrm{PCV}$, policarbonatos, mezclas y poliestireno (cuadro IV). El 11.4\% de los productos fueron fabricados con PCV y contenían ftalatos en concentraciones que fueron desde trazas hasta $67.0 \%$ del peso

\section{Cuadro II \\ Potenciales fuentes de exposición a ftalatos. ToluCA, México, 1999}

\begin{tabular}{lrc} 
& \multicolumn{3}{l}{ Frecuencia* } \\
\cline { 2 - 4 } Fuentes & No. & $\%$ \\
& 139 & 69.8 \\
\hline Utensilios de plástico para comer & 73 & 36.7 \\
\hline Recipientes de plástico para guardar alimentos & 44 & 22.1 \\
\hline Recipientes plásticos para calentar comida en microondas & 60 & 30.1 \\
\hline Papel plástico para conservar alimentos en refrigeración & 32 & 16.0 \\
\hline Compra de alimentos empacados en plástico & & \\
* El número de fuentes sobrepasa el número de niños encuestados debido \\
a que éstos pueden estar expuestos a más de una
\end{tabular}

salud pública de méxico / vol.46, no.6, noviembre-diciembre de 2004 


\section{Cuadro III}

\section{CoMpaRACIÓN BIVARIADA DE CARACTERÍSTICAS SOCIODEMOGRÁFICAS Y SOCIOECONÓMICAS ENTRE LOS NIÑOS QUE}

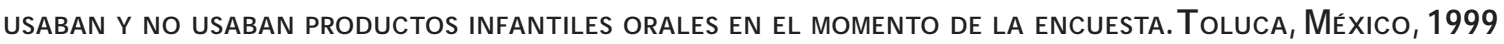

\begin{tabular}{|c|c|c|c|c|c|}
\hline Variable & Categoría & Usan & No usan & $\chi^{2}$ & Valor de $p$ \\
\hline Edad de la madre & Media (años) & 27.4 & 28.7 & & 0.24 \\
\hline Edad del niño & Media en meses & 16.6 & 24 & & $<0.001$ \\
\hline \multirow[t]{2}{*}{ Género } & Femenino & 73 & 23 & 5.19 & 0.03 \\
\hline & Masculino & 91 & 12 & & \\
\hline Peso del niño & Media (g) & 10376 & 12007 & & 0.002 \\
\hline \multirow[t]{4}{*}{ Estrato socioeconómico } & Medio & 97 & 28 & 5.37 & 0.02 \\
\hline & 0 tro & 67 & 7 & & \\
\hline & Bajo & 63 & 7 & 4.29 & 0.04 \\
\hline & 0 tro & 101 & 28 & & \\
\hline \multirow[t]{2}{*}{ Ocupación de la madre } & Ama de casa & 117 & 26 & 0.12 & 0.72 \\
\hline & 0 tro & 47 & 9 & & \\
\hline \multirow[t]{2}{*}{ Cuidado del niño } & Mamá & 128 & 27 & 0.01 & 0.91 \\
\hline & 0 tro* & 36 & 8 & & \\
\hline \multirow[t]{2}{*}{ Antecedentes de lactancia materna } & Sí & 103 & 76 & 0.18 & 0.67 \\
\hline & No & 17 & 3 & & \\
\hline
\end{tabular}

*A buelos, hermanos, guardería

Cuadro IV

Materiales identificados en PROductos infantiles DE USO ORAL OBTENIDOS DURANTE LA ENCUESTA. TolucA, MÉxico, 1999

\begin{tabular}{lcc} 
Material & $n$ & $\%$ \\
Silicona & 91 & 44.8 \\
\hline Polipropileno & 30 & 14.8 \\
\hline Polietileno & 26 & 12.8 \\
\hline Policloruro de vinilo (PCV) & 18 & 8.9 \\
\hline Policarbonato & 14 & 6.9 \\
\hline Mezclas & 8 & 3.9 \\
\hline Látex & 6 & 2.9 \\
\hline Otro no identificado & 6 & 3.0 \\
\hline Poliestireno & 4 & 2.0 \\
\hline Total & 203 & 100.0
\end{tabular}

Nota: El número de materiales identificado sobrepasa el de objetos obtenidos en la encuesta debido a que estos últimos pueden contener dos 0 tres piezas fabricadas con diferentes materiales

total del producto. El ftalato más utilizado fue el DEHP ftalato de (di- 2 etyl exilo). En el cuadro V se observa que los productos que contenían PCV y ftalatos como plastificantes fueron mordederas, chupones, mamilas y juguetes que algunas mamás reportaron que el niño se llevaba a la boca para chuparlo o morderlo. En el caso de las mordederas las concentraciones variaron desde 11.0 hasta $62.0 \%$ del peso total. En el caso de las mamilas utilizadas para la alimentación de los niños las concentraciones encontradas fueron desde trazas hasta $15.0 \%$. Los chupones de vasos entrenadores variaron su concentración desde trazas hasta casi 5.0\% y en dos de los juguetes blandos se encontraron concentraciones de 67.0 y $52.0 \%$, respectivamente. Los valores de la última columna de este cuadro muestran las concentraciones de ftalatos que fueron extraídas mediante hexano.

Las concentraciones difieren de acuerdo con el tiempo de uso del producto, lavado, succión, calor, etcétera, así que para calcular la difusión se consideraron aquellas más altas que utilizan los fabricantes como lo es 40 PCV / 60 DEHP. El promedio de concentración final de ftalato de DEHP en saliva humana in vivo fue de 1.5 con una DE de 0.27 y su difusión de 6.04 $\mu \mathrm{g} / \mathrm{cm}^{2} / \mathrm{h}$. La ingesta diaria promedio por esta fuente de exposición para el grupo de edad en estudio, con las especificaciones que se describieron en la sección de métodos, se estimó en $13.9 \mu \mathrm{g} / \mathrm{kg} /$ día, IC 95\% (9.18, 18.89).

\section{Discusión}

En esta encuesta los niños menores de tres años de edad, especialmente los varones menores de 18 meses 


\section{Cuadro V \\ Determinaciones cualitativas Y CUANTITATIVAS de fTALATOS EN PROdUCtOS Fabricados Con PCV mediante análisis infrarrojo y Cromatografía de gaSes.Toluca, México, 1999}

\begin{tabular}{|c|c|c|c|c|c|}
\hline $\begin{array}{l}\text { Descripción } \\
\text { (tipo de producto) }\end{array}$ & $\begin{array}{l}\text { Número } \\
\text { de piezas }\end{array}$ & Composición & $\begin{array}{c}\text { Tipo } \\
\text { de ftalato }\end{array}$ & $\begin{array}{c}\% \text { de ftalato } \\
\text { por cromatografía de gases }\end{array}$ & $\begin{array}{c}\text { Concentración de ftalato } \\
\text { en } \mathrm{mg} / \mathrm{cm}^{3}\end{array}$ \\
\hline Solvente & & Hexano. RA & No & 0 & \\
\hline PCV puro & & PCV & No & 0 & \\
\hline Mordedera & 1 & PCV & DEHP & 10.6 & 72.9 \\
\hline Vaso entrenador & 1 & PCV & DEHP/EHF & 0.1 & 1.0 \\
\hline Mordedera & 1 & PCV & DEPH & 10.0 & 73.3 \\
\hline Juguete suave & 1 & PCV & DEHP & 67.2 & 466.6 \\
\hline Taza entrenadora & 1 & PCV & DEHP & 0.9 & 6.5 \\
\hline Mordedera & 1 & PCV & DEHP & 30.4 & 210.8 \\
\hline Chupón de vaso entrenador & 1 & PCV & DEHP & 1.4 & 9.4 \\
\hline Chupón pacificador & 1 & PCV & DEHP & 0.10 & 0.69 \\
\hline Juguete suave & 1 & PCV & DEHP & 51.9 & 360.2 \\
\hline Mamila & 1 & PCV & $\mathrm{DnBP}$ & 10.0 & 69.4 \\
\hline Mamila & 1 & PCV & DEHP & 2.0 & 14.0 \\
\hline Mordedera & 1 & PCV & DEHP & 62.3 & 432.9 \\
\hline Mamila & 1 & PCV & DEHP & 14.9 & 103.6 \\
\hline Mamila & 1 & PCV & DEHP & 0.2 & 1.0 \\
\hline Mamila & 1 & PCV & DEHP & 2.2 & 15.1 \\
\hline Chupón de vaso entrenador & 1 & PCV & DEHP & 4.7 & 33.8 \\
\hline Mamila & 1 & PCV & DEHP & 10.1 & 69.9 \\
\hline Mamila & 1 & PCV & DEHP & 0.9 & 6.0 \\
\hline Mamila & 1 & PCV & $\mathrm{DnBP}$ & 10.0 & 182.9 \\
\hline
\end{tabular}

N ota: Se analizaron los datos por cromatografía de gases únicamente de los artículos que resultaron estar elaborados con PCV y de aquellos de los cuales se tenía duda de su material de fabricación

PCV: policloruro de vinilo

pertenecientes al estrato socioeconómico bajo utilizaron con mayor frecuencia productos infantiles de uso oral y, por lo tanto, tienen una mayor probabilidad de exposición a los ftalatos. Estas observaciones se han encontrado en otros estudios. ${ }^{8}$

Parece ser que el uso de los productos infantiles diseñados para ser llevados a la boca en la población estudiada tiene que ver con características tanto propias de los niños como la edad y género, como con variables de tipo social como el estrato al que pertenecen. Biológicamente existe un periodo de la vida en el que el niño, ya sea por su alimentación o por la etapa de su desarrollo, tiende a usar este tipo de productos. La prevalencia de uso de los productos motivo del estudio tiene que ver más con la alimentación que con la necesidad de chupar o morder, ya que de acuerdo con nuestras observaciones los niños hacían mayor uso de productos relacionados con la alimentación, como se observa en el cuadro I; el tiempo promedio de uso diario fue de media hora.
El cuidado de los niños es básicamente proporcionado por las madres, las cuales en promedio han terminado su educación secundaria. Esta información es importante porque todos las recomendaciones resultado de este estudio tendrán que ser dirigidas a este segmento de la población, por lo que deberán buscarse los mecanismos y vías más adecuadas; por ejemplo, parte de las recomendaciones de las instituciones de salud se dirigirían a las madres en sus domicilios, ya que la mayoría de éstas son amas de casa que mencionaron encargarse personalmente del cuidado de sus hijos pequeños, mediante personal de campo que realiza actividades en programas permanentes o intensivos; los mensajes tendrían que ser diseñados por los responsables de la comunicación de riesgos y deberían contener información sobre los productos seguros que los niños pueden llevarse a la boca para chupar, morder o alimentarse.

El mayor uso de los productos en el estrato social bajo probablemente se deba a que estos niños compen- 
san sus carencias materiales chupando este tipo de productos. Entre las mamás de este grupo social tendrán que dirigirse con mayor fuerza los mensajes o recomendaciones en torno al uso de productos motivo del estudio, ya que los hijos de estas mujeres fueron los principales expuestos al uso pues los llevan a sus bocas por más tiempo y las madres lactan menos a sus hijos.

El uso de productos infantiles de uso oral es frecuente entre los niños menores de tres años de edad. Sin embargo, como puede observarse, los tipos de productos que más habitualmente utilizan estos niños son los relacionados con la alimentación, es decir, mamilas de biberón. Lo que significa que nuestra atención deberá dirigirse a este tipo de productos y en los de marcas más conocidas. El ftalato más frecuentemente utilizado en los productos fabricados con PCV fue el DEHP y sus concentraciones variaron entre trazas hasta más de $60 \%$ del peso total del producto; sin embargo, es importante recordar que estos productos eran usados y las cantidades de ftalatos utilizados en su fabricación están de acuerdo con la flexibilidad requerida, generalmente superiores a $50 \%$, por lo que consideramos que las concentraciones encontradas subestiman las proporciones originales. Por otra parte, los esfuerzos por comparar productos nuevos similares a los captados en la encuesta en cuanto al tipo de ftalato y su contenido no fueron satisfactorios, ya que a partir de 1999 dejó de utilizarse el PCV para fabricar productos de "marca" para uso oral. No obstante, previo a la encuesta los fabricantes e importadores en México de este tipo de productos enviaron voluntariamente al laboratorio de la UAEM muestras de mordederas, chupones y juguetes blandos donde se encontraron concentraciones de ftalatos superiores a $50 \%$.

Entre los productos obtenidos de la encuesta se encontró $11.0 \%$ de éstos fabricados con PCV plastificados principalmente con DEHP. La atención de las autoridades de salud tendrá que dirigirse al monitoreo, a través de su muestreo y análisis sistemático en el laboratorio, del tipo de material utilizado para fabricar los más frecuentemente usados, y principalmente los de aquellas marcas no líderes o de fabricación casera. Además, tendrán que vigilarse no sólo los productos importados para asegurarse que no se trata de productos retirados de los mercados de origen por contener estas sustancias, aquellos de dudosa procedencia.

A la luz del conocimiento actual existe incertidumbre sobre los efectos de los ftalatos basados en evidencias de daños a la salud en animales. Se ha observado que los ftalatos y sus metabolitos producen disrupción sexual masculina en varios sitios del aparato reproductor y con diferentes grados de severidad. La testosterona juega el papel central de los efectos antiandrogénicos de los ftalatos. ${ }^{5}$ Esta información es además importante, ya que si consideramos estos efectos, se tendrá que tener un especial cuidado con los niños, pues de acuerdo con nuestros resultados son ellos quienes utilizan más este tipo de productos que las niñas.

Actualmente tiende a reconocerse que algunos ftalatos como el DEHP tienen poca actividad como cancerígeno, y algunos organismos internacionales han solicitado su cambio de categoría a una de menor riesgo. ${ }^{12}$ Sin embargo, hay que continuar otros estudios que evalúen los efectos de estas sustancias y sus metabolitos en virtud de que, como se mencionó, se han encontrado efectos reproductivos a través de mecanismos de disrupción endocrina en varias etapas críticas del desarrollo sexual masculino en animales al comportarse los ftalatos como sustancias antiandrogénicas. ${ }^{13-16}$ Además, se han observado efectos reproductivos directos sobre mujeres embarazadas, manifestados como complicaciones del embarazo como preeclampsia e hiperemesis, principalmente, ${ }^{17}$ y disminución de la función pulmonar. ${ }^{18}$

Es importante considerar además de los productos infantiles de uso oral otro tipo de exposiciones. Nuestro estudio captó información sobre este aspecto. La principal fuente de exposición para la población general son los alimentos contaminados con el empaque. ${ }^{19}$ En 1995, en un estudio realizado en Canadá demostraron la cantidad de ftalatos encontrados en diversos alimentos y bebidas, además de que el uso de microondas acelera la migración de los ftalatos de los recipientes plásticos a los alimentos. En la población estudiada exploramos el uso de trastes plásticos para comer, de amplio uso en nuestro medio, además de las costumbres en relación con el guardado de los alimentos, uso de microondas y compra de alimentos empacados en plástico, los que deberán ser considerados para acercarnos a la verdadera exposición a estas sustancias. Es necesario mencionar que no todos los productos fabricados con plástico contienen ftalatos, pero existe una mayor probabilidad de exposición a éstos si se tiene preferencia por utensilios o productos que los contienen. Generalmente, cuando los productos plásticos son fabricados con PCV el plastificante utilizado es un ftalato, sin embargo existen otros tipos de plásticos (polímeros) que no requieren de la adicción de ftalatos como los listados en el cuadro IV. Para la identificación del tipo de material (polímero) con el que fueron fabricados los productos se utilizó análisis de infrarrojo.

Otro aspecto importante es la velocidad con la que los ftalatos se desprenden de los productos plásticos..$^{11,20}$ 
El Comité Científico de Toxicidad, Ecotoxicidad y Ambiente de EUA ha propuesto límites aceptables para diversos ftalatos. ${ }^{11,20}$ A partir de los datos de frecuencia y tiempo del uso de los objetos de interés y los niveles detectados en los mismos y su migración se pudo estimar hipotéticamente los niveles de exposición a los cuales se encuentran sometidos este grupo de niños y que se calculó la ingesta diaria hasta en $13.9 \mu \mathrm{g} / \mathrm{kg}$. de peso/día. La cuantificación de ftalatos de los productos de la encuesta fue realizada con otros propósitos y las técnicas de extracción no permiten la cuantificación de la ingesta. Para esta estimación, como se mencionó previamente, la velocidad de difusión fue de $6.0 \mu \mathrm{g} / \mathrm{cm}^{2} / \mathrm{h}$, lo cual indica que la migración del DEHP es muy lenta y no rebasa el valor límite aceptable de $10.0 \mu \mathrm{g} / \mathrm{cm} 2 / \mathrm{h}$ recomendado por US CSTEE y la dosis diaria proveniente de estas fuentes fue estimada en $13.9 \mu \mathrm{g} / \mathrm{kg}$ de peso/día. Sin embargo, consideramos que la ingesta calculada en este estudio sólo considera la exposición por estos productos; para calcular la exposición total se deben considerar otras fuentes como los alimentos, particularmente los lácteos, por ello deberán buscarse otro tipo de marcadores de exposición más eficientes por lo que proponemos la utilización de biomarcadores en orina, medida a través de las concentraciones de metabolitos primarios de ftalatos, esta técnica fue propuesta por el Centro de Control de Enfermedades de EUA (CDC, por sus siglas en inglés) en el año 2000 y ha sido adaptada para su uso en el Laboratorio de Química Instrumental, de la UAEM. ${ }^{21}$

\section{Referencias}

1. N ational Toxicology Program. (1982a) Carcinogenesis bioassay of di (2etylhexyl)phthalate(CAS N 0. 103-23-1) in F344 rats and B6C 3F1 mice (feed study).Tech. Rep. Ser.212. Research Triangle Park (N C), USA: National toxicology Program; 1982.

2. N ational Toxicology Program (N TP) (1982a) carcinogenesis bioassay of di(2etylhexyl)phthalate(CAS N 0.117-81-7) in F344 rats and B6C 3F1 mice (feed study) Tech. Rep. Ser.217. Research Triangle Park (NC), USA: National Toxicology Program; 1982.

3. N ational Toxicology Program, $\mathrm{N}$ ational Institute of Environmental Health Sciences. Final Report on the Reproductive Toxicity of Di(nButyl) Phthalate (CAS N 0 84-74-2) in Sprague-D aw ley Rats. N ational Technical Information Service (N TIS), U.S. Department of Commerce; Springfield (VA): N TIS Publication N 0. 92-111996.
4. Harrison PT, Holmes P, Humfrey CD. Reproductive health in humans and widelife:Are adverse trends associated with environmental chemical exposure? Sci Total Environ 1997;205:97-106.

5. Mylchreest E, Foster PM. Antiandrogenic effects of di(n-Butyl) phthalate on male reproductive development:A nonreceptor-mediated mechanism. C IIT Activities1998;18:1-10.

6. G reenpeace. PVC toys and phthalates. England: G reenpeace Press; 1997.

7. Marín ML, López J, Sánchez A,Vilaplana J, Jiménez A. A nalysis of potentially toxic phthalate plasticizers used in toy manufacturing. Bull Environ Contam Toxicol 1998;60:68-73.

8. Babich MA.The risk of chronic toxicity associated with exposure to disononyl phthalate (DIN P) in children's products. Report of U.S. Consumer Product Safety.W ashington, DC: CPSC; 1998.

9. Treybal RE. 0 peraciones de transferencia de masa. 20 edición. México, DF: MC Graw Hill; 1988.

10. Corea-Téllez KS. Difusión de ftalatos provenientes de plastificados de policloruro de vinilo (PCV), en agua y saliva (tesis). Toluca, Estado de México: Universidad Autónoma del Estado de México; 2002.

11. European Scientific Committee on Toxicity, Ecotoxicity, and the Environment. 0 pinion on phthalate migration from soft PVC toys and childcare articles. 0 pinión expressed at the $6^{\text {th }}$ (CSTEE Plenary Meeting; 1998 nov 26-27; Brussels. Disponible en: http://europa.eu.int/comm/ food/fs/sc/sct/out19_en.html [2001 July 3].

12. D oull J, C attley R, Elcombe C, Lake BG, Swenberg J,W ilkinson C et al. Cancer risk assessment of $\mathrm{Di}(2$-ethylhexyl) phthalate:A pplication of the N ew U.S. EPA Risk Assessment Guidelines. Regul Toxicol Pharmacol 1999:29:327-357.

13. Thomas JA, N orthup SJ. Toxicity and metabolism of monoetylhexyl phthalate and dietylhexyl phthalate:A survey of recent literature.J Toxicol Environ Health 1982;9:141-152.

14. Foster PMD.Assesing the effects of chemicals on male reproduction: Lessons learnes fron di-n-butyl phthalate. C IIT Activities 1997;17(9):1-8. 15. Parmar D, Srivastava SP, Singh GB,Seth PK. Testicular toxicity of di(2ethylhexyl) phthalate in developing rats. Vet Hum Toxicol 1995;37: 310-311.

16. $N$ ational Toxicology Program, $C$ enter for the Evaluation of Risks to Human Reproduction; ntp-cerhr Expert Panel Report on Di(2ethylhexyl)phthalate. ntp-cerhr-dehp-00. Research Triangle Park (N C): 2000: http://cerhr.niehs.nih.gov/news/D EHP- final.pdf [2001 julio 19]. 17. Tavacova S, Little R, Balavaeva L. Maternal exposure to phthalates and complications of pregnancy. Epidemiology 1999; suppl 10:127. 18. Hoppin JA, U Imer R, London SJ. 2004. Phthalate exposure and pulmonary function. Environ Health Perspect: [0 $\mathrm{nline}$ ] D isponible en: http://ehp.niehs.nih.gov/docs/2004/6564/abstract.html.

19. Page BD, Lacroix GM.The occurrence of phthalate ester and di-2 etylhexyl adipate plasticizers in Canadian packaging and food sampled in 1985-89 a survey. Food Addit Contam 1995;12:129-151.

20. EU Scientific Committee for Toxicity, Ecotoxicity and the Environment (CSTEE). Phthalate migration from soft PVC toys and child-care articles. 0 pinion expressed at the CSTEE third plenary meeting. 199824 A pril. Brussels. Disponible en: http://europa.eu.int/ comm/health/ph_risk/committees/sct/documents/out45_en. pdf. 21. Blount BC, Milgram KE, Silva MJ, Malek N A, Reidy JA, N eedham LL et al. Q uantitative detection of eight phthalate metabolites in human urine using HPLC-APCI-MS/MS. Anal Chem 2000;72:4127-4134. 